\title{
Synthesis of silver nanowires by using tetrabutyl ammonium dibromochloride as the auxiliary for low-haze flexible transparent conductive film
}

Ximin Yuan, Hongwei Yang*, Yuxiu Li, Yao Li, Yunxiu Chao, Jialin Chen, Li Chen

State Key Laboratory of Advanced Technologies for Comprehensive Utilization of

Platinum Metals, Kunming Institute of Precious Metals, 650106 Kunming, People's

Republic of China

\section{Supporting Information}

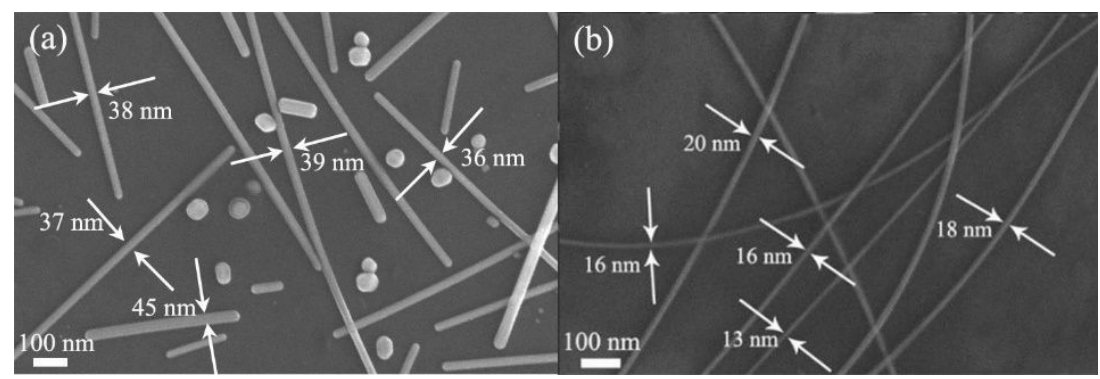

Figure S1. (a) FE-SEM image of AgNWs prepared by $\mathrm{NaCl}$. Except for use of $\mathrm{NaCl}$ as an auxiliary agent, the other preparation conditions are the same as in this work. (b) FE-SEM image of AgNWs prepared by TBADBC. 

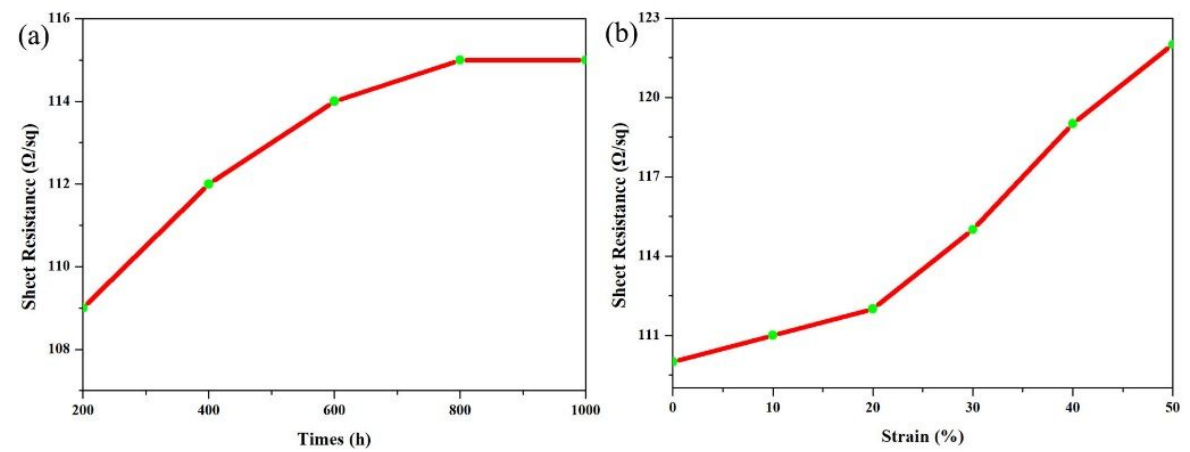

Figure S2. (a) Anti-oxidation capability of AgNWs film. (b) Sheet resistance of AgNWs film as a function of strain. 